\title{
Padrões clínicos de acne em mulheres de diferentes faixas etárias
}

\author{
Acne in women: Clinical patterns in different age-groups
}

\author{
Juliano Vilaverde Schmitt ${ }^{1}$ \\ Hélio Amante Miot ${ }^{3}$
}

Paula Yoshiko Masuda ${ }^{2}$

Resumo: FundAMENTOS: Acne é dermatose comum que acomete ambos os gêneros e todas as faixas etárias. Mulheres apresentam diferentes padrões clínicos da doença, além de frequente persistência da acne após a adolescência.

OвJETIVO: Analisar características clínicas e epidemiológicas associadas às diferentes faixas etárias acometidas por acne feminina.

MÉTODOS: Estudo transversal envolvendo mulheres com diagnóstico de acne, atendidas em ambulatório de dermatologia geral. Variáveis relacionadas à doença e às pacientes foram avaliadas com o emprego de questionário padronizado.

Resultados: Avaliaram-se 103 pacientes, cuja idade média na época da consulta foi $21,7 \pm 7,3$ anos. Definiram-se dois subgrupos com idade de corte de 21 anos e idades médias de 15,8 $\pm 2,3$ e 28,0 \pm 5,1 anos. Houve correlação entre a duração do quadro e a idade das pacientes na consulta $(\mathrm{R}=0,7)$. Observaram-se diferenças entre os grupos nas frequências de uso de contraceptivo oral combinado (OR = $48,1)$, lesões no colo $(\mathrm{OR}=11,6)$, lesões no dorso $(\mathrm{OR}=0,2)$, predominância na topografia superior da face $(\mathrm{OR}=0,1)$ e idade de início das lesões $(\mathrm{OR}=1,8)$. No grupo de mulheres adultas, $80 \%$ relataram início do quadro antes dos 20 anos.

CONCLUSÕES: Identificaram-se padrões clínicos cronológicos e topográficos que caracterizaram a acne feminina em diferentes faixas etárias, alertando para a importância da abordagem diagnóstica e terapêutica individualizada.

Palavras-chave: Acne vulgar; Dermatologia; Estudos transversais; Hiperandrogenismo

\begin{abstract}
BACKGROUND: Acne is a frequent skin disease that occurs in both sexes and all age-groups. Women present several clinical disease patterns; moreover, persistence after adolescence is common.

OвjестілE: To analyze clinical and epidemiological characteristics associated with different age-groups affected by acne in women.

METHODS: Cross-sectional study involving female patients diagnosed with acne, at a general dermatology outpatient clinic. Variables related to disease and patients were assessed through a standardized questionnaire. RESULTS: One hundred and three women were assessed. The average age of patients at the time of the consultation was $21.7 \pm 7.3$ years. Two groups were defined (cut-off age of 21 years), with means of $15.8 \pm$ 2.3 and $28.0 \pm 5.1$ years. There was correlation between disease duration and current age $(\mathrm{R}=0.7)$. There were group differences among frequencies of covariables: combined oral contraceptive (OR=48.1), lesions located on upper chest $(\mathrm{OR}=11.6)$, lesions on upper dorsum $(\mathrm{OR}=0.2)$, predominance on upper half of face $(\mathrm{OR}=0.1)$ and age at disease onset $(\mathrm{OR}=1.8)$. Among adult women, $80 \%$ reported acne onset before 20 years of age.

Conclusion: Chronologic and topographic patterns of female acne in different age-groups were defined, reinforcing the importance of an individualized diagnostic and therapeutic approach.

Keywords: Acne Vulgaris; Cross-Sectional Studies; Dermatology; Hyperandrogenism
\end{abstract}

Recebido em 16.01.2009

Aprovado pelo Conselho Consultivo e aceito para publicação em 29.05.09.

* Trabalho realizado na Fundação Pró-Hansen - Curitiba (PR), Brasil.

Conflito de interesse: Nenhum / Conflict of interest: None

Suporte financeiro: Nenhum / Financial funding: None

Dermatologista da Fundação Pró-Hansen - Curitiba (PR), Brasil.

Médica estagiária da Fundação Pró-Hansen - Curitiba (PR), Brasil.

Professor-assistente doutor do Departamento de Dermatologia e Radioterapia da Faculdade de Medicina de Botucatu da Universidade Estadual Paulista (Unesp) - Botucatu (SP), Brasil.

(C)2009 by Anais Brasileiros de Dermatologia 


\section{INTRODUÇÃO}

Acne é dermatose frequente que totaliza cerca de $14 \%$ das consultas dermatológicas no Brasil e acomete ambos os sexos, diferentes etnias e todas as faixas etárias, com predominância nas três primeiras décadas de vida. Apresenta características morfológicas diversas de acordo com a etiologia e a idade de manifestação. No entanto, há significante sobreposição entre essas manifestações clínicas e as categorias etárias envolvidas. ${ }^{1,2}$

Alterações hormonais da puberdade estão quase sempre relacionadas com o início da acne vulgar típica, e os adolescentes do sexo masculino são os mais frequentemente $\mathrm{e}$ intensamente afetados. Maturação adrenal e desenvolvimento gonadal levam a produção de andrógenos e subsequente aumento das glândulas sebáceas, culminando com a erupção de acne nessa faixa etária. ${ }^{3-5}$

Sugere-se que a intensidade da acne se relacione melhor com o estágio puberal do que com a idade cronológica; mesmo assim, a maioria dos pacientes masculinos espera a regressão da acne entre os $20 \mathrm{e}$ 25 anos. Em contraste, mulheres podem continuar com o problema durante a vida adulta, mesmo após os 40 anos de idade. ${ }^{6}$

Quando avaliados os estudos observacionais, Collier et al. identificaram prevalência de queixas de acne em 51\% das mulheres entre 20 e 29 anos. Goulden et al. revisaram os registros de 200 pacientes com acne entre 25 e 55 anos, verificando que $76 \%$ eram mulheres e que, em $82 \%$ dos casos, havia persistência da acne da adolescência. Porém, Poli et al. observaram apenas 59\% com esse perfil e constataram que a prevalência só se reduzia significativamente após os 45 anos. ${ }^{8,9}$

A acne feminina pós-adolescência pode ser dividida em persistente, a qual representa uma continuidade do quadro relacionado à puberdade, e acne de início tardio, que se inicia após os 25 anos de idade. Acne do mento é uma forma intrigante que ocorre no período pré-menstrual em mulheres maduras, enquanto na acne esporádica há o súbito desenvolvimento das lesões na vida adulta, sem razão aparente. Clinicamente, essas formas de acne podem diferir da dos adolescentes porque tendem a ser mais inflamatórias, com menos comedões. Além disso, as lesões são mais comumente localizadas ao redor da boca, mento e linha da mandíbula. ${ }^{10}$

Uso de cosméticos, alterações hormonais e nutricionais, coexistência de hirsutismo, dermatite seborreica, alopecia, irregularidade menstrual ou aspectos psicoevolutivos são elementos aventados na explicação dos diferentes padrões clínicos e de história natural da acne em diferentes grupos populacionais. ${ }^{8,11-20}$
Há carência de pesquisas epidemiológicas que comparem aspectos clínicos associados à acne feminina juvenil e adulta. O presente estudo objetiva analisar as características clínicas e epidemiológicas associadas às diferentes faixas etárias acometidas por acne nessa população.

\section{MATERIAL E MÉTODOS}

Realizou-se estudo transversal envolvendo todos os pacientes femininos atendidos no ambulatório de dermatologia geral da Fundação Pró-Hansen, situada em Curitiba-PR, durante o período de janeiro a dezembro de 2008, com diagnóstico de acne. As pacientes foram contactadas por telefone e entrevistadas pela autora (PYM), por meio de questionário padronizado.

Os prontuários foram revisados com a finalidade de exclusão das pacientes com diagnóstico explícito de acne secundária a medicamentos ou cosméticos.

A variável dependente analisada foi a faixa etária das pacientes no momento da consulta. As covariáveis exploradas foram: história familiar de acne, uso de cosméticos faciais por mais que três dias por semana, uso de contraceptivo oral, padrão menstrual anormal, oleosidade da pele, dermatite seborreica do couro cabeludo, predominância topográfica (acima ou abaixo da metade da face, colo, dorso, ombros) e padrão morfológico (pápulas ou comedões).

Variáveis categóricas foram representadas como frequências percentuais em cada grupo (adulto e juvenil), sendo as proporções comparadas pelos testes de Fisher e qui-quadrado. Variáveis contínuas foram representadas pela média e desvio-padrão e comparadas pelo teste de Mann-Withney; sua correlação linear foi medida pelo coeficiente de Spearman. Moda e mediana foram empregadas na descrição de amostras não paramétricas. A normalidade das distribuições foi estimada pelo teste de Lilliefors. ${ }^{21}$

As variáveis foram testadas por um modelo de regressão logística múltipla condicional e reduzidas a partir de um algoritmo (stepwise backward), com critério de manutenção de $\mathrm{p}<0,3 \mathrm{em}$ cada passo.

A associação entre as variáveis foi estimada a partir da odds ratio (OR) e de seu intervalo de confiança de $95 \%$ (IC 95\%).

A idade de corte para classificação como acne adulta e juvenil foi estimada pela média das idades de melhor discriminação entre as covariáveis, empregando a curva ROC.

A tabulação e a análise dos dados foram realizadas pelo software MS Excel 2003 e pelo software SPSS. ${ }^{17}$ Considerou-se significativo valor de $\mathrm{p}<0,05$ bicaudal. ${ }^{22}$ 


\section{RESULTADOS}

Foram atendidas 138 mulheres com o diagnóstico de acne no período referido. Excluindo-se os casos de acne secundária e considerando-se a perda amostral por impossibilidade de contato telefônico, foram incluídas 103 (74,6\%) pacientes no estudo.

A idade média ( \pm desvio-padrão) das pacientes na época da consulta foi $21,7 \pm 7,3$ anos; o histograma etário (Gráfico 1), porém, identifica dois picos modais (15 e 27 anos). Para a aplicação dos testes de comparação entre proporções, a análise discriminatória pela curva ROC definiu dois grupos a partir da idade de corte de 21 anos, gerando duas amostras com médias de $15,8 \pm 2,3$ e $28,0 \pm 5,1$ anos, compostas por 53 e 50 indivíduos.

A idade média do início dos sintomas foi de 14,3 \pm 4,1 anos (Gráfico 2), e a mediana da duração do quadro foi de cinco anos (Gráfico 3). Observou-se forte correlação positiva entre a duração do quadro e a idade das pacientes na consulta (RSpearman $=0,74$; $\mathrm{p}<0,01$ ), o que não ocorreu com a idade de início dos sintomas ou com a menarca.

A comparação bivariada entre os grupos etários quanto às variáveis independentes está descrita na tabela 1. Destaquem-se as diferenças nas frequências de uso do contraceptivo oral combinado, lesões no dorso, predominância nas topografias superior e inferior (de acordo com o grupo) e idade de início das lesões.

A partir da análise multivariada, constatou-se associação significativa dos grupos etários com a idade de início do quadro, uso de contraceptivo oral combinado, predominância de lesões na parte superior da face, presença de lesões no dorso e presença de lesões no colo (Tabela 2).

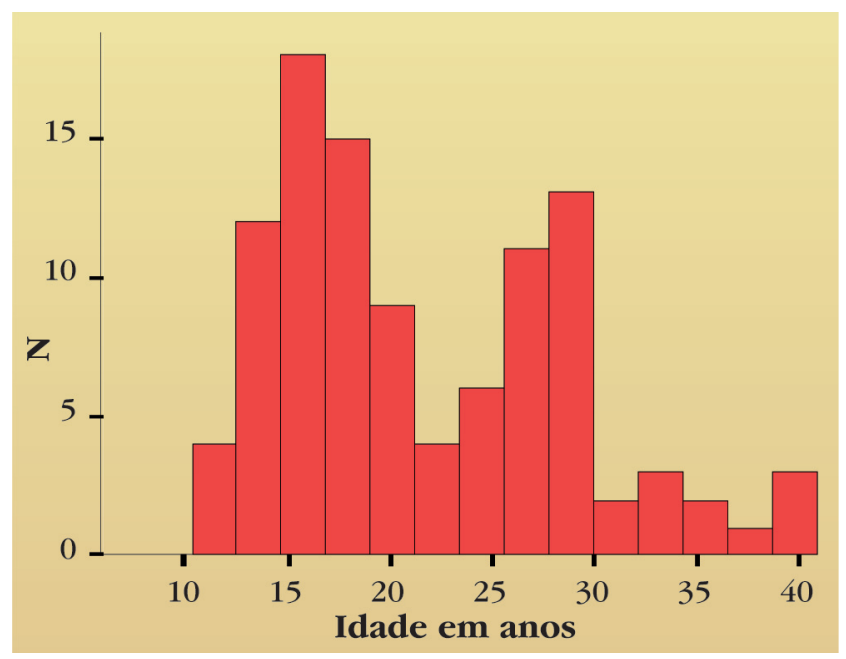

Gráfico 1: Histograma etário das pacientes na data da consulta

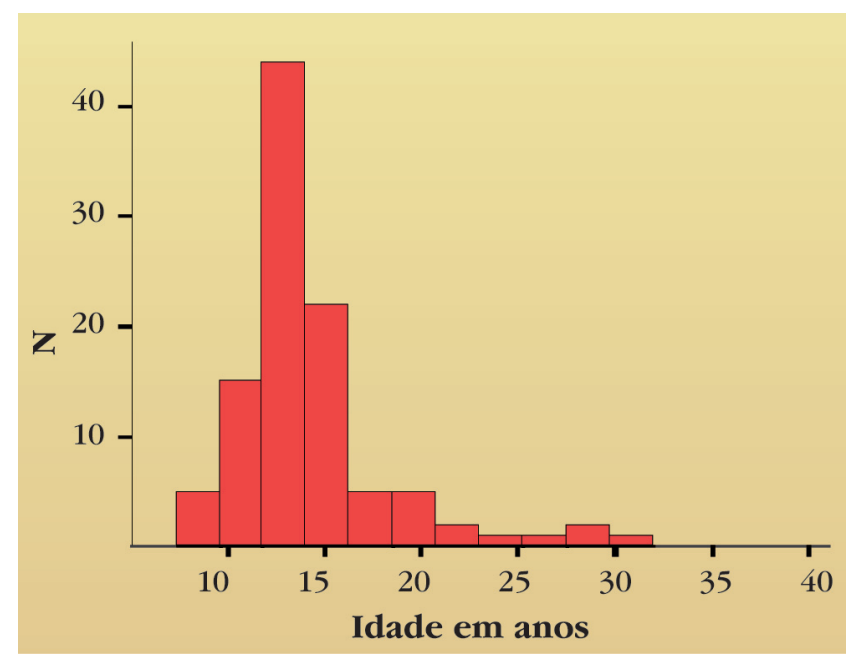

Gráfico 2: Histograma das idades de início dos sintomas

\section{DISCUSSÃ̃}

Os dados apresentados permitem identificar duas populações diferentes de pacientes femininas portadoras de acne quando avaliadas sob o aspecto etário, o que reforça a observação clínica da existência de entidades com certa independência no aspecto clínico, e provavelmente fisiopatológico, já descritas como a acne da adolescente (vulgar) e a acne da mulher adulta. ${ }^{4,10}$

Os resultados sugerem que a idade discriminatória entre esses grupos giraria em torno dos 21 anos, corroborando algumas publicações, mas estando abaixo do limite de 25 anos, o mais utilizado em estudos prévios. Essa pequena discrepância pode refletir aspectos culturais e geográficos da população estudada ou até aspectos metodológicos usados para definir esse corte. ${ }^{7,10,11,15}$

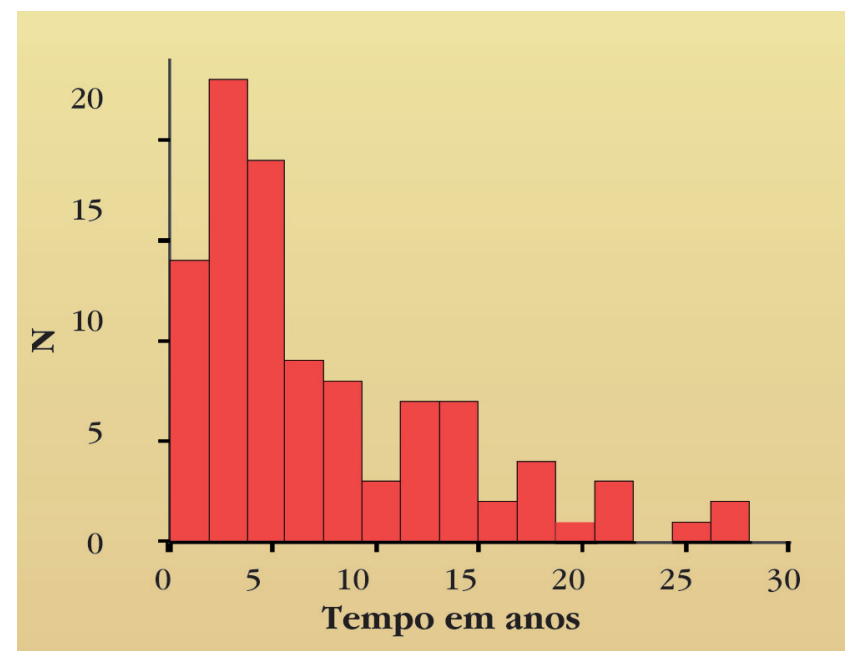

Gráfico 3: Histograma do tempo de duração do quadro 
TABela 1: Análise comparativa bivariada das variáveis independentes de acordo com o grupo etário

\begin{tabular}{|c|c|c|c|c|c|c|}
\hline \multirow[t]{2}{*}{ Variáveis } & \multicolumn{2}{|c|}{$<21$ anos } & \multicolumn{2}{|c|}{$\geq 21$ anos } & Odds Ratio & \multirow[t]{2}{*}{$\mathbf{P}$} \\
\hline & $\mathbf{N}$. & $\%$ & $\mathbf{N}$. & $\%$ & (IC 95\%) & \\
\hline História familiar de acne & 34 & 68 & 27 & 58,7 & $0,67 \quad 0,29-1,54$ & $0,39 *$ \\
\hline Uso de cosméticos & 11 & 20,8 & 12 & 24 & $1,21 \quad 0,48-3,05$ & $0,69 *$ \\
\hline Contraceptivo oral combinado & 2 & 3,8 & 16 & 32 & $12 \quad 2,59-55,57$ & $0,00 *$ \\
\hline Contraceptivo com progestágeno & 1 & 1,9 & 2 & 4 & $2,170,19-24,67$ & $0,61 * *$ \\
\hline Alterações menstruais & 18 & 35,3 & 16 & 32 & $0,860,38-1,97$ & $0,73^{*}$ \\
\hline Dermatite seborreica do couro cabeludo & 17 & 32,1 & 16 & 32 & $1 \quad 0,44-2,28$ & $0,99 *$ \\
\hline Pele oleosa & 46 & 86,8 & 41 & 82 & $0,69 \quad 0,24-2,03$ & $0,50 *$ \\
\hline Acne no colo & 10 & 18,9 & 10 & 20 & $1,08 \quad 0,40-2,85$ & $0,88^{*}$ \\
\hline Acne no dorso & 35 & 66 & 23 & 46 & $0,44 \quad 0,20-0,97$ & $0,04 *$ \\
\hline Acne nos ombros & 6 & 11,3 & 7 & 14 & $1,280,40-4,09$ & $0,68^{*}$ \\
\hline Predominância de pápulas & 34 & 64,2 & 39 & 78 & $1,98 \quad 0,83-4,75$ & $0,12 *$ \\
\hline Predominância de comedões & 35 & 66 & 33 & 66 & $1,00 \quad 0,44-2,26$ & $1,00 *$ \\
\hline Predominância de lesões abaixo da linha média da face & 16 & 30,2 & 27 & 54 & $2,71 \quad 1,21-6,09$ & $0,01 *$ \\
\hline Predominância de lesões acima da linha média da face & 48 & 90,6 & 35 & 70 & $0,24 \quad 0,08-0,73$ & $0,01 *$ \\
\hline Idade de surgimento das lesões (média \pm desvio-padão) & 12,7 & $\pm 2,3$ & 16,1 & $\pm 4,8$ & - & $0,00 * * *$ \\
\hline
\end{tabular}

* Teste do qui-quadrado; ** Teste exato de Fisher; *** Teste de Mann-Withney

Algumas variáveis classicamente associadas às mulheres adultas com acne, como o uso de cosméticos, irregularidade menstrual e obesidade, não foram corroboradas por este estudo, demonstrando que as características topográficas da acne devem exercer melhor capacidade classificatória, quando ponderada a faixa etária envolvida. Da mesma forma, a história familiar não se mostrou elemento significativamente proeminente entre os grupos, o que não afasta a possibilidade de que ambas as formas tenham ascendência familiar na mesma intensidade; entretanto, o viés de memória não pode ser desconsiderado nesse caso.

Não houve maior frequência no predomínio de pápulas e lesões inflamatórias nas mulheres adultas, contrariando algumas observações prévias quanto ao perfil clínico destas. A não associação com o tipo de lesão, se retencional ou inflamatória, pode dever-se à falha das entrevistadas em identificarem as respectivas características morfológicas. Por outro lado, a entrevistadora não percebeu dificuldade na realização dos inquéritos. ${ }^{8,10,11,23}$

Houve diferença significativa na idade de início da acne entre os grupos. Mesmo assim, 80\% das mulheres do grupo adulto relataram o início das lesões ainda na adolescência, ratificando observações prévias de que a maioria dos casos de acne de mulheres adultas pode corresponder a persistência do quadro juvenil. A alta correlação entre a duração do quadro e a idade da paciente na consulta reforça essa hipótese. ,9,24 $^{8,24}$

As características clínicas que mais parecem determinar os grupos etários de acne entre as mulheres, além da idade de início do quadro, referem-se à topografia das lesões: observou-se predomínio de aco-

TABela 2: Modelo reduzido de regressão logística múltipla condicional para análise das variáveis independentes de acordo com o grupo etário

\begin{tabular}{|c|c|c|c|}
\hline \multirow{2}{*}{$\begin{array}{l}\text { Variáveis do modelo } \\
\text { Contraceptivo oral combinado }\end{array}$} & \multirow{2}{*}{$\begin{array}{l}\mathbf{p} \\
0,00\end{array}$} & \multicolumn{2}{|c|}{ Odds Ratio (IC 95\%) } \\
\hline & & 48,05 & $6,03-383,07$ \\
\hline Alterações menstruais & 0,17 & 0,41 & $0,11-1,47$ \\
\hline Acne no colo & 0,01 & 11,57 & $1,92-69,69$ \\
\hline Acne no dorso & 0,01 & 0,18 & $0,04-0,71$ \\
\hline Predominância de lesões acima da linha média da face & 0,02 & 0,14 & $0,03-0,73$ \\
\hline Pele oleosa & 0,22 & 3,05 & $0,50-18,46$ \\
\hline Predominância de comedões & 0,19 & 2,52 & $0,64-9,98$ \\
\hline Idade de surgimento das lesões & 0,00 & 1,75 & $1,28-2,38$ \\
\hline Constante & 0,00 & 0,00 & \\
\hline
\end{tabular}

$\mathrm{p}$ (modelo completo) < 0,01; Verossimilhança $(-2 \log )=74,42 ;$ Prova de Hosmer-Lemeshow: $\mathrm{p}=0,15$; Classificação correta: 77,6\% 
metimento na metade superior da face, além do dorso, nas pacientes mais jovens, enquanto nas adultas houve maior ocorrência de lesões no colo, além da face.

$\mathrm{O}$ uso de contraceptivo oral se associou fortemente ao grupo etário, já que se espera um aumento da sua frequência de uso entre as mulheres adultas; entretanto, sua interferência nos quadros acneicos não pode ser desprezada, o que soma mais uma razão para compor o modelo multivariado final, ajustando os resultados.

O presente estudo não contemplou a análise de parâmetros laboratoriais de hiperandrogenismo, apesar de a acne representar uma manifestação dessa condição clínica. Na condição de um estudo observacional em que grande parte das mulheres se revelou usuária de pílula anticoncepcional, a fidelidade das estimativas de certos androgênios plasmáticos poderia estar comprometida. Outro elemento importante ocorre junto à controvérsia de que acne persistente de forma isolada seja manifestação de hiperandrogenismo. Cibula e colaboradores não demonstraram correlação entre a gravidade da acne e qualquer sinal clínico ou marcador laboratorial de hiperandrogenismo. Não obstante, observou-se que mulheres com quadros mais intensos apresentavam menores índices de testosterona livre, menos hirsutismo e níveis maiores de SHBG (sexual hormone-binding globulin). ${ }^{18}$ Elementos externos, como leite e derivados, calor, filtros solares e períodos de estresse psicológico, também são descritos como agravantes dos quadros de acne, desfavorecendo o hiperandrogenismo como causa de todos os quadros de acne da mulher adulta e interferindo no seu tratamento. ${ }^{13,14,25}$
A concepção psicológica evolucionista da acne poderia explicar as diferenças adquiridas no padrão de acometimento de mulheres e homens no decorrer da formação da espécie. Segundo Bloom, a persistência de acne na vida da mulher corroboraria a indicação de um sinal ligado à adolescência que retardaria o acasalamento precoce, permitindo à mulher ganhar maturidade e maior condição de cuidado para a sobrevivência da cria. Apesar da dificuldade de comprovação de hipóteses evolutivas, não se encontram argumentos fisiológicos que justifiquem a persistência de acne em mulheres adultas, até próximo do fim da sua vida reprodutiva, e a seleção sexual poderia ser uma explicação plausível. ${ }^{19}$

Os resultados deste trabalho levam a duas considerações distintas: uma em que ocorreriam mudanças no perfil clínico de uma mesma doença em decorrência das modificações fisiológicas causadas pela idade e outra em que assumimos estar diante de duas condições nosológicas separadas.

Novos estudos que englobem amostras de outras populações, investiguem aspectos fisiopatológicos e sirvam de comparação para elucidar as bases para os achados deste estudo devem ser conduzidos.

\section{CONCLUSÃO}

Foram identificados padrões clínicos cronológicos e topográficos que favoreceram a caracterização da acne feminina em diferentes faixas etárias (juvenil e adulta), alertando para a importância da abordagem diagnóstica e terapêutica individualizada da acne nesses grupos. 


\section{REFERÊNCIAS}

1. Sociedade Brasileira de Dermatologia. Perfil nosológico das consultas dermatológicas no Brasil. An. Bras Dermatol. 2006;81:549-58.

2. Dreno B, Poli F. Epidemiology of acne. Dermatology. 2003;206:7-10.

3. Hassun KM. Acne: etiopatogenia. An Bras Dermatol. 2000;75:7-15.

4. Steiner D. Acne na mulher. Rev Bras Med. 2002;59:135-9.

5. Costa A, Alchorne MMA, Goldschmidt MCB. Fatores etiopatogênicos da acne vulgar. An Bras Dermatol. 2008;83:451-9.

6. Lucky AW, Biro FM, Simbartl LA, Morrison JA, Sorg NW. Predictors of severity of acne vulgaris in young adolescent girls: results of a five-year longitudinal study. J Pediatr. 1997;130:30-9.

7. Collier CN, Harper JC, Cafardi JA, Cantrell WC, Wang W, Foster KW, et al. The prevalence of acne in adults 20 years and older. J Am Acad Dermatol. 2008;58:56-9.

8. Goulden V, Clark SM, Cunliffe WJ. Post-adolescent acne: a review of clinical features. Br J Dermatol. 1997; 136:66-70.

9. Poli F, Dreno B, Verschoore M. An epidemiological study of acne in female adults: results of a survey conducted in France. J Eur Acad Dermatol Venereol. 2001; 15:541-5.

10. Marks R. Acne and its management beyond the age of 35 years. Am J Clin Dermatol. 2004;5:459-62.

11. Kligman AM. Postadolescent acne in women. Cutis. 1991;48:75-7.

12. Cordain L, Lindeberg S, Hurtado M, Hill K, Eaton SB, Brand-Miller J. Acne vulgaris: a disease of Western civilization. Arch Dermatol. 2002;138:1584-90.

13. Chiu A, Chon SY, Kimball AB. The response of skin disease to stress: changes in the severity of acne vulgaris as affected by examination stress. Arch Dermatol. 2003;139:897-900.

14. Adebamowo CA, Spiegelman D, Berkey CS, Danby FW, Rockett HH, Colditz GA, Willett WC, Holmes MD. Milk consumption and acne in teenaged boys. J Am Acad Dermatol. 2008;58:787-93.

15. Seirafi H, Farnaghi F, Vasheghani-Farahani A, Alirezaie NS, Esfahanian F, Firooz A, et al. Assessment of androgens in women with adult-onset acne. Int J Dermatol. 2007; 46:1188-91.
16. Cappel M, Mauger D, Thiboutot D. Correlation between serum levels of insulin-like growth factor 1, dehydroepiandrosterone sulfate, and dihydrotestosterone and acne lesion counts in adult women. Arch Dermatol. 2005; 141:333-8.

17. Lucky AW. Quantitative documentation of a premenstrual flare of facial acne in adult women. Arch Dermatol. 2004; 140:423-4.

18. Cibula D, Hill M, Vohradnikova O, Kuzel D, Fanta M, Zivny $\mathrm{J}$. The role of androgens in determining acne severity in adult women. Br J Dermatol. 2000; 143:399-404.

19. Bloom DF. Is acne really a disease?: a theory of acne as an evolutionarily significant, high-order psychoneuroimmune interaction timed to cortical development with a crucial role in mate choice. Med Hypotheses. 2004;62:462-9.

20. Vexiau P, Baspeyras M, Chaspoux C, Foin N, Allaert FA, Abramovici Y. [Acne in adult women: data from a national study on the relationship between type of acne and markers of clinical hyperandrogenism.]. Ann Dermatol Venereol. 2002; 129:174-8.

21. Henderson AR. Testing experimental data for univariate normality. Clin Chim Acta. 2006;366:112-29.

22. SPSS 17.0 for Windows [computer program] [cited 2008 Dez 25]. Statistical Package for Social Science (SPSS). Release Version 17.0.1. Chicago (IL): SPSS Incorporation; 2008. Available from: http:// www.spss.com.

23. Menon C, Gipson K, Bowe WP, Hoffstad OJ, Margolis DJ. Validity of subject self-report for acne. Dermatology. 2008;217:164-8.

24. Dumont-Wallon G, Dréno B. [Specificity of acne in women older than 25 years]. Presse Med. 2008;37:585-91.

25. Sampaio SAP, Bagatin E. Experiência de 65 anos no tratamento da acne e de 26 anos com isotretinoína oral. An. Bras. Dermatol. 2008;83:361-7.

ENDEREÇO PARA CORRESPONDÊNCIA / MAILING ADDRESS: Juliano Vilaverde Schmitt.

Rua Fernando Amaro, $n^{\circ} 1.116$ - Cristo Rei 80050020 Curitiba PR

Tel./fax: (41) 3024-2757-3263-2757.

E-mail:julivs@gmail.com

Como citar este artigo/How to cite this article: Schmitt JV, Masuda PY, Miot HA. Padrões clínicos de acne em mulheres de diferentes faixas etárias. An Bras Dermatol. 2009;84(4):349-54. 